\title{
Developing a Registry of Healthcare-Associated Infections at Intensive Care Units in West China: Study Rationale and Patient Characteristics
}

This article was published in the following Dove Press journal: Clinical Epidemiology

\author{
Wen Wang ${ }^{1, *}$ \\ Shichao Zhu ${ }^{2, *}$ \\ Qiao $\mathrm{He}^{\prime}$ \\ Rui Zhang ${ }^{3}$ \\ Yan Kang ${ }^{4}$ \\ Mingqi Wang' \\ Kang Zou' \\ Zhiyong Zong ${ }^{2,5}, *$ \\ Xin Sun ${ }^{1, *}$ \\ 'Chinese Evidence-Based Medicine \\ Center and CREAT Group, West China \\ Hospital, Sichuan University, Chengdu \\ 61004I, People's Republic of China; \\ ${ }^{2}$ Department of Infection Control, West \\ China Hospital of Sichuan University, \\ Chengdu, People's Republic of China; \\ ${ }^{3}$ Information Center, West China \\ Hospital, Sichuan University, Chengdu, \\ People's Republic of China; ${ }^{4}$ Intensive \\ Care Unit, West China Hospital of \\ Sichuan University, Chengdu, People's \\ Republic of China; ${ }^{5}$ Center of Infection \\ Diseases, West China Hospital of Sichuan \\ University, Chengdu, People's Republic of \\ China
}

*These authors contributed equally to this work

\begin{abstract}
Purpose: Limited evidence is available to support the management of healthcare-associated infections (HAIs) in intensive care units (ICUs). Establishing a registry with complete and accurate information is urgently needed. The West China Hospital system has the most complete and largest data system for HAI in the ICU setting in China. By linking a multidimensional database, we developed a registry of HAI in ICU.
\end{abstract}

Methods: The ICU-HAI registry was established using a multi-source database that included electronic medical record (EMR), ICU system and ICU-HAI system in the West China Hospital healthcare system. Patients who were admitted to ICUs between 1 April 2015 and 30 March 2018 were included and data were extracted based on pre-designed, standardized data forms. We achieved the linkage of the three databases using a unique patient identification code, and cleaned the data based on standardized variable dictionaries and cleaning rules. We evaluated the quality of the registry through data verification and assessment of the quality of key variables.

Results: In total, 23, 062 patients were included. The ICU mortality and hospital mortality were $5.4 \%$ and $5.5 \%$ respectively. A total of 855 patients developed ICU-HAIs, 1540 patients developed ventilator-associated events (VAE), and 171 patients developed possible ventilator-associated pneumonia (PVAP). Quality assessment showed that the accuracy of data extraction and linkage was $100 \%$. Furthermore, $98 \%$ of all patients had at least one important laboratory tests performed, and the median number of tests performed was 4 to 5 per admission.

Conclusion: A unique registry for HAIs in the ICU setting was successfully established, which contains complete and accurate information for all patients in the ICU. The registry, linked from multiple data sources, provides unique research insights into the management of HAIs in the ICU setting in China.

Keywords: healthcare-associated infection, intensive care units, registry

\section{Introduction}

Chinese Evidence-Based Medicine Center West China Hospital, Sichuan University,

37 Guo Xue Xiang, Chengdu, Sichuan 61004I, People's Republic of China Email sunx79@hotmail.com

Zhiyong Zong

Department of Infection Control, West

China Hospital, Sichuan University,

Chengdu, Sichuan 61004I, People's

Republic of China

Email zongzhiyong@gmail.com
Healthcare-associated infections (HAIs), an infections acquired in healthcare settings, have become a major concern for hospitals, especially in intensive care units (ICUs). ${ }^{1-3}$ Due to impaired host defense, underlying diseases, multiple invasive devices, and multiple drug-resistant organisms associated with the use of broadspectrum antimicrobial agents, HAIs are common in the ICU setting. The proportion of patients with HAIs usually account for over $30 \%$ of all ICU admissions. ${ }^{3}$ The Extended Prevalence of Infection in Intensive Care (EPIC II) study, including 14,414 patients from 1, 265 ICUs, reported $51 \%$ of patients with infections. ${ }^{2}$ The 
ICU patients with HAIs may have a poorer prognosis that includes prolonged hospital stays, and increased healthcare costs and mortality. ${ }^{2,4,5}$ The EPIC II study suggested that compared with patients without infections, the ICU mortality was more than two times higher among those with HAIs. $^{2}$ Compared with patients without central lineassociated bloodstream infection (CLABSI), ICU mortality was increased by $30 \%$ among ICU patients with CLABSI, $^{4}$ and costs were increased by $\$ 39,000$ per episode. $^{5}$

The management of HAIs is a major challenge for healthcare worldwide. Several authorities, such as the World Health Organization (WHO), the US Center for Disease Control (CDC), Society for Healthcare Epidemiology of America (SHEA) and Infectious Diseases Society of America (IDSA) have published guidelines regarding the prevention and control of HAIs. ${ }^{5-8}$ Many recommendations were, however, based on low-quality evidence. For instance, in the guideline updated in 2014, SHEA recommended 16 strategies to prevent CLABSI, among which, only two were based on a moderate quality of evidence and the others were based on a low quality of evidence. ${ }^{5}$ In another example, due to subjective and unspecific diagnostic criteria, traditional ventilator-associated pneumonia (VAP) surveillance using clinical criteria have important limitations in practice. Thus, in 2013, the US CDC developed new surveillance criteria for ventilator-associated events (VAE), which aimed to capture all complications leading to deterioration among mechanically ventilated patients. ${ }^{9,10}$ Nevertheless, current strategies for the prevention of VAE were with little support from sound evidence. The available studies about the newly-defined VAE typically have small sample sizes and report only a few events. ${ }^{11-13}$

The lack of high-quality evidence is primarily because the data were either unavailable or inadequate to answer questions of interest. The completeness and accuracy of data are often two important issues for generating robust evidence for HAIs in the ICU. For instance, traditional HAI surveillance exclusively collects data of patients with HAIs, and such healthcare data, as diagnostic and treatment information, are usually unavailable. ${ }^{14-16}$ This limits the evaluation of interventional strategies for HAIs. Therefore, establishing a registry with complete and accurate information of all admissions using multidimensional data sources is essential to address critical issues about HAIs. ${ }^{17,18}$
West China Hospital is a major healthcare system in west China, which consists of three independent healthcare organizations (University Campus, Wenjiang Hospital, and Jinjiang Hospital) (Figure S1) ${ }^{19}$ In total, the healthcare system has 4300 beds. Consistently ranked in the second place among all hospitals in China, the healthcare system provides tertiary care for the population of Sichuan (of over 80 million) and other provinces. In 2016, 5.3 million hospital visits and 223,000 discharges were recorded. ${ }^{19}$

The ICUs at the healthcare system were initially established in 1992, and have become a national critical care center in west China. Since 2015, the healthcare system has been comprised of six ICUs and 172 beds, with more than 8000 ICU inpatients annually. To improve management of HAIs in the ICU setting, the healthcare facilities developed a specific data system for ICU services in 2015 , in addition to the routine electronic medical records (EMRs), and a prospective surveillance system for HAIs. All data in the three data systems (EMR, ICU, and HAI surveillance) were collected and stored using a unique patient identifier, which was linked to a unique social identity (ID).

By connecting such data sources to develop a linked registry, important information can be obtained to address many issues regarding HAIs in the ICU setting. To the best of our knowledge, the HAI surveillance system has now become the most complete and largest data system for HAIs in the ICU setting in China, and is unique for the routine surveillance of VAE across the country. This paper elaborated our efforts to develop the registry of HAIs in the ICU setting within the West China Hospital system. We also presented the patient baseline.

\section{Methods}

This study was approved by the Ethical Committee of West China Hospital in August 2018 (WCH2018-409). It was conducted using routinely collected health data, which were obtained for administrative and clinical purposes without specific a priori research goals; therefore, patient consent was waived. We used de-identified data which contained a significant level of protection against the release of personal information to outside entities.

\section{Data Sources and Linkage}

To acquire complete information addressing important issues regarding HAIs in the ICU setting, we developed an ICU-HAI registry by integrating data from the ICUHAI system, the ICU system, and EMR at West China 
Table I Key Details About the ICU-HAI Registry

\begin{tabular}{|l|l|l|l|}
\hline & HAI System & EMR System & ICU System \\
\hline $\begin{array}{l}\text { Methods of } \\
\text { data collection }\end{array}$ & $\begin{array}{l}\text { Prospective surveillance by } \\
\text { infection control practitioners. } \\
\text { Basic information were } \\
\text { extracted from EMR }\end{array}$ & $\begin{array}{l}\text { Routinely collected health data from clinical } \\
\text { process }\end{array}$ & Critical care information recorded by nurse \\
\hline $\begin{array}{l}\text { Information } \\
\text { recorded }\end{array}$ & $\begin{array}{l}\text { Catheterization information, } \\
\text { hospital-acquired } \\
\text { infection, prevention and } \\
\text { control measures }\end{array}$ & $\begin{array}{l}\text { Demographics, diagnosis, laboratory and } \\
\text { microbiology tests, prescription, operation, } \\
\text { admissions records, discharge summaries } \\
\text { and costs }\end{array}$ & $\begin{array}{l}\text { Shift records, nurse notes, vital signs, life } \\
\text { supports, checklists }\end{array}$ \\
\hline $\begin{array}{l}\text { Frequency of } \\
\text { data collection }\end{array}$ & $\begin{array}{l}\text { Prospective surveillance data } \\
\text { were collected every day; basic } \\
\text { information were extracted in } \\
\text { real time }\end{array}$ & $\begin{array}{l}\text { Same as actual clinical process: admissions } \\
\text { records were recorded within 24 hrs of } \\
\text { admission; }\end{array}$ & $\begin{array}{l}\text { Vital signs, life supports, intake and output } \\
\text { volume were recorded every hour; shift records } \\
\text { were recorded every } 8 \text { hrs; APACHE score } \\
\text { were recorded within 24 hrs of admission }\end{array}$ \\
\hline Follow-up time & During ICU stay & During hospital stay & During ICU stay \\
\hline $\begin{array}{l}\text { Department } \\
\text { coverage }\end{array}$ & Six ICUs of WCH & Both ICU and non-ICU & Six ICUs of WCH \\
\hline
\end{tabular}

Hospital Healthcare System. The linkage was performed by a unique and encoded identifier for each patient. The registry included data from six ICUs, including the general ICU (GICU), surgical ICU (SICU), neurological ICU (NICU), respiratory ICU (RICU), thoracic surgery ICU (TICU) and pediatric ICU (PICU). Detailed descriptions of the ICU-HAI multi-source database are summarized in Table 1 and Figure 1.

The ICU-HAI system is a prospective surveillance system for HAIs that has been fully adopted by the healthcare system since 2015. A team of three infection control practitioners actively collected information regarding catheterization, the duration of catheterization, and prevention and control measures, and judged suspected ICU-HAI cases according to The US CDC-National Healthcare Safety Network (NHSN) criteria. ${ }^{20,21}$ Basic information, such as age, sex, and ICU types, was retrieved from the EMR system in real-time. To ensure the accuracy of data, logical checks were automatically conducted. Furthermore, manual checks were performed by the infection control practitioners through a monthly chart review. One important feature of the ICU-HAI system was its coverage of all patients admitted to ICUs, regardless of the incidence of HAIs has occurred. This contrasts with other ICU-HAI systems, which only record information of patients with HAIs. The second important feature of the ICU-HAI system was its unique establishment of routine VAE surveillance. Each year, the ICU-HAI system undertake the monitoring of more than 8000 person-time of HAI and 5000 cases of VAE.

The EMR system initially developed in 2008, includes the Health Information System (HIS), Laboratory Information System (LIS), and Picture Archiving and Communication System (PACS). The EMR contains detailed information regarding patient demographics, diagnoses, laboratory and microbiological tests, treatments, notes, and cost. ${ }^{22}$ Most of the data are stored in structured or semi-structured formats, including demographics, diagnoses, tests, prescriptions, and cost. In 2018, more than $99 \%$ of records had International Classification of Diseases (ICD) coding, and validation of the ICD-10 code showed an accuracy of $88 \%$ (internal reports). The EMR has been previously used for clinical research. ${ }^{4,22}$

The ICU data system, independent of the EMR, was initially developed in 2012 and was fully adopted in all ICUs in 2015. The data were electronically recorded by well-trained specialty nurses, and contained information regarding vital signs, life support, nurses' notes, risk assessments, and ICU-HAI related checklists (i.e., central line catheter checklist and disinfection checklist), which were not routinely collected in other intensive care settings in China.

\section{Study Population}

The ICU-HAI registry included patients that were admitted to any of the six ICUs at West China Hospital 


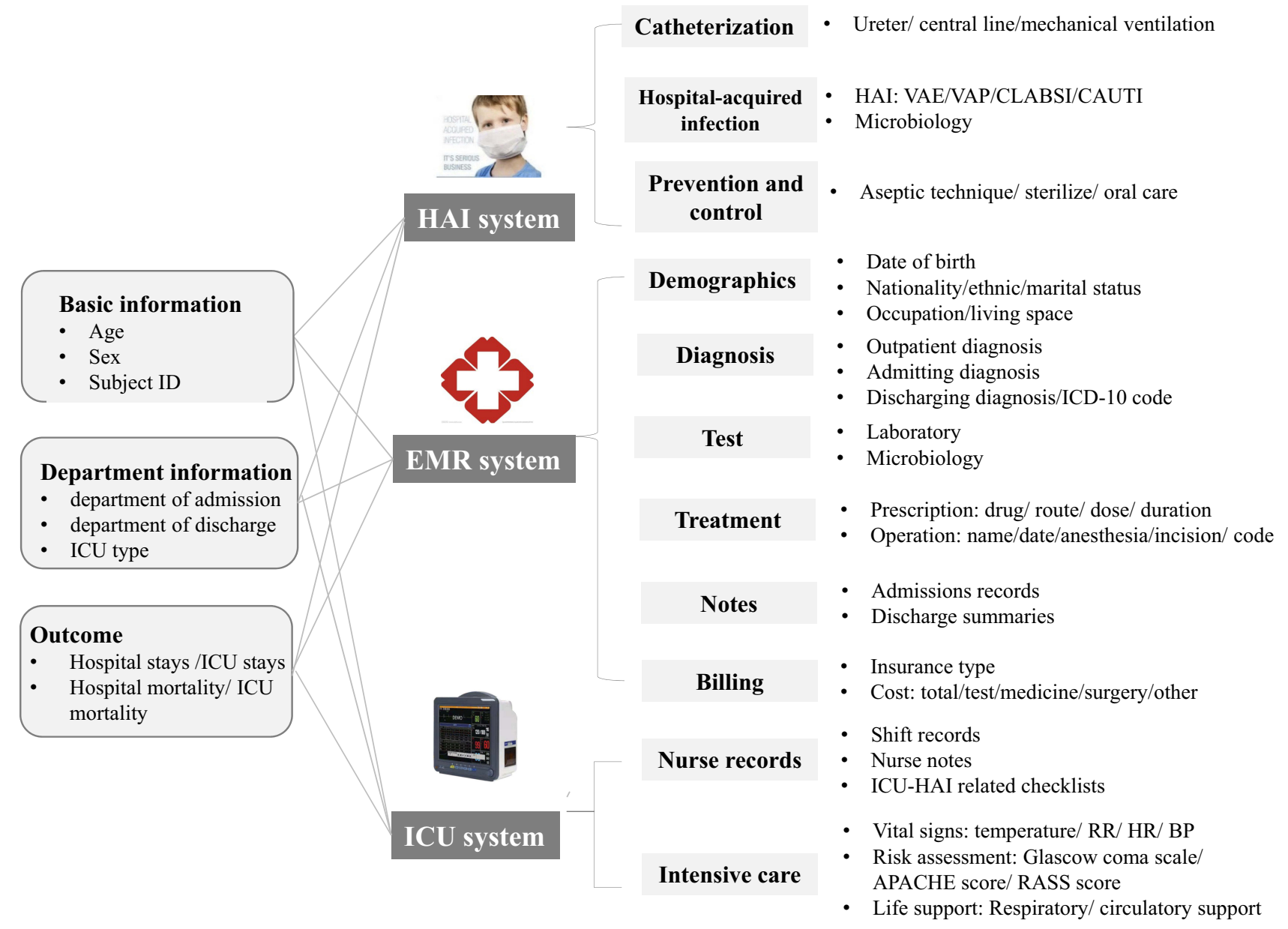

Figure I Data sources for establishing the ICU-HAI registry.

between 1 April 2015 and 30 March 2018. We excluded patients if they met any of the following criteria: 1) incomplete information on date of birth, sex and discharged diagnosis; 2) non-Chinese nationality; 3) absence of a valid patient identifier; or 4) extremely long ICU stay and abnormal bill due to medical disputes.

\section{Data Collection and Cleaning}

The process of developing the ICU-HAI registry is summarized in Figure 2. A multidisciplinary research team was developed for the registry, including epidemiologists, statisticians, and experts in critical care medicine, infection control, and information technology.

The information experts extracted data from the EMR, ICU, and ICU-HAI systems, using the pre-designed, standardized data forms. The extracted data were then deidentified by information specialists, through removing identifier data elements, including patient name, telephone number, address, and identity card number.
The data were stored in TXT or EXCEL format, and linked using a unique identifier developed by the information department at the healthcare system. The unique identifiers included the patient ID (unique to each patient) and admission ID (for each unique admission). To further identify a unique admission to an ICU, we developed an ICU ID, based on information regarding the admission ID, date of admission to the ICU and date of discharge from ICU.

We cleaned the data using transparent and prespecified rules, including the development of variable dictionaries, standardization of medical texts, and approaches to missing data and outliers. We established variable dictionaries for basic information, diagnoses (ICD-10 coding and free terms); operations (invasive operation or non-invasive operation); tests (laboratory tests, microbial tests, and test samples); intensive care (vital signs and life support); prescriptions (drug dictionary and administration route) and ICU-HAI 


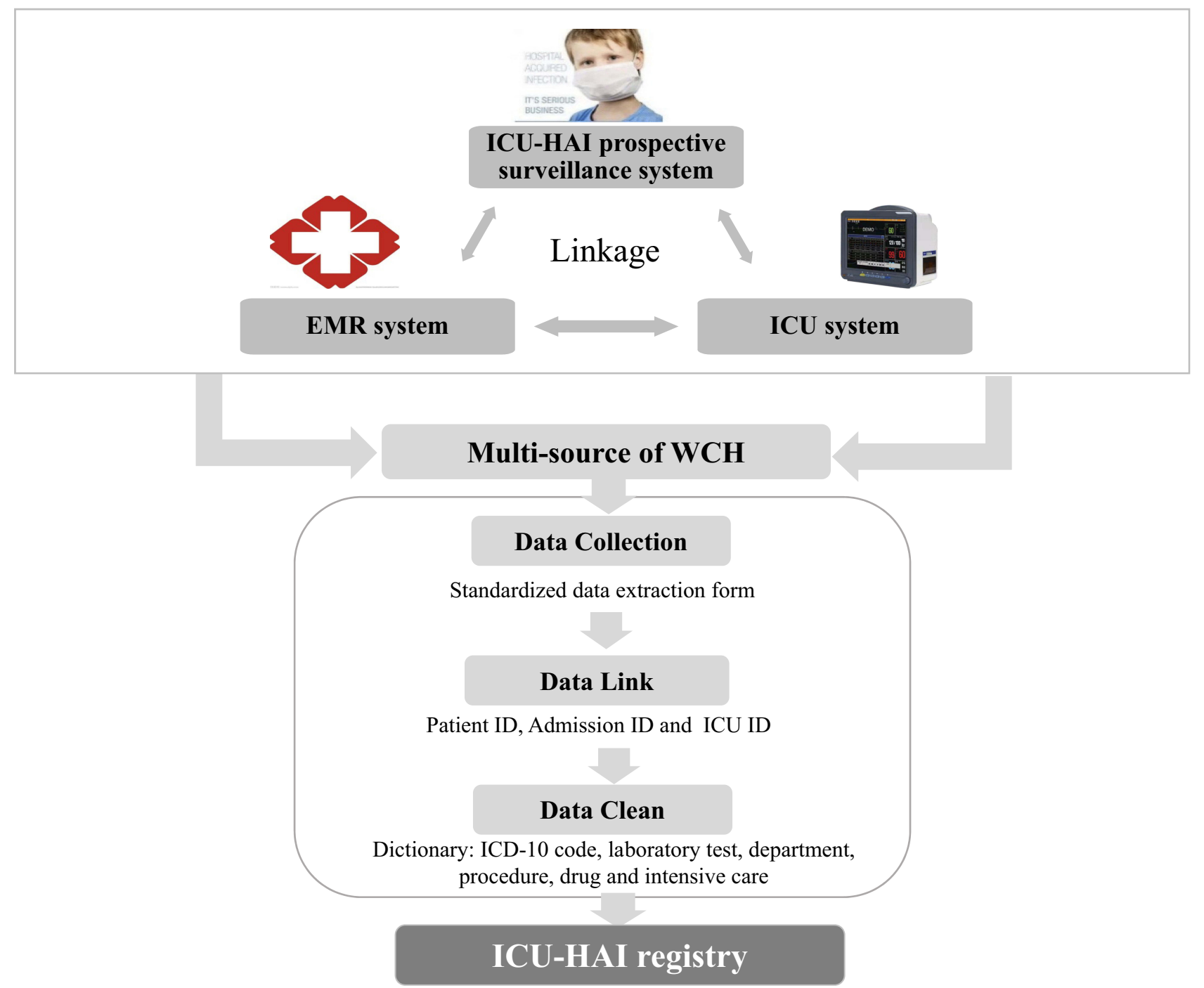

Figure 2 Process of registry development.

information (catheterization, infection site, and pathogenic bacteria). The primary ICD-10 codes of discharge diagnosis are listed in Table S1.

\section{Quality Control}

To assess the quality and reliability of the ICU-HAI registry, we reviewed 240 randomly selected medical charts. Two investigators independently reviewed data regarding basic information, laboratory tests, prescribing information, intensive care, and catheterization, and checked the extracted and linked records against the original data. We also evaluated the proportion of missing data and outliers for key variables, including sociodemographics, laboratory tests and vital signs. To validate the accuracy of VAE, 158 medical charts of the possible ventilator-associated pneumonia (PVAP) cases were reviewed and evaluated by two experienced infection control practitioners.

\section{Results}

A total of 23, 062 patients were included in the ICU-HAI registry, who accounted for 23, 847 ICU admissions between 1 April 2015 and 30 March 2018. We collected 110 GB of data with 245,311,294 original records, which contained 492 variables with detailed information regarding the clinical care of patients, and HAI prevention and control.

Characteristics and Clinical Outcomes of Patients in the ICU-HAI Registry

Of the 23, 062 ICU patients, 8158 (34.2\%) were from Chengdu municipal city, 
$12,454(52.2 \%)$ from other cities in Sichuan province, and 2049 (8.6\%) from other provinces. A total of 4543 patients (19.7\%) were admitted to the GICU, 4343 (18.8\%) to the SICU, $2033(8.8 \%)$ to the NICU, 1338 (5.8\%) to the RICU, 6407 (27.8\%) to the TICU and 5183 (22.5\%) to the PICU. Furthermore, 13,711 (57.5\%) patients were male, $4623(19.4 \%)$ patients were 18 years of age or younger, and $6425(26.9 \%)$ were 65 years of age or older. The median length of ICU stay was 4 days (interquartile range: 3-8 days) and the median length of hospital stay was 16 days (12-24 days). In total, 1244 (5.4\%) deaths occurred during the ICU stay, and 1321 (5.5\%) deaths during hospitalization. The top five diagnoses at discharge were valvulopathy $(3551,14.9 \%)$, heart septal defectsatrial $(2175,9.1 \%)$, cherebral hemorrhage $(1669,7.0 \%)$, pneumonia (1452, $6.1 \%$ ) and aortic dissection (894, 3.7\%) (Table 2).

Compared with patients admitted to other ICUs, a higher proportion of male patients $(907,67.8 \%)$ and patients aged 65 years or older $(737,55.1 \%)$ were admitted to the RICU. The proportion of patients with a diagnosis of valvulopathy $(3445,53.8 \%)$ and aortic dissection $(694,10.8 \%)$ at discharge was higher in the TICU than in other ICUs. The proportion of patients with a diagnosis of heart septal defectsatrial was higher $(1666,32.1 \%)$ in the PICU, and the proportion of patients with pneumonia was higher in the RICU (824, 61.6\%). The median durations of ICU stay (11 days) and hospital stay (19 days) were longer in the RICU compared with other units. The ICU mortality and hospital mortality were $24.1 \%$ (322 deaths) and $26.0 \%$ (348 deaths), respectively (Table 2).

In the ICU-HAI registry, 855 patients accounted for 972 cases of ICU-HAIs and a total of 199,992 patient days (at a rate of 4.86 cases per 1000 patient days). The rates of HAIs varied across different ICUs, and patients admitted to the NICU had the highest rate of HAIs ( 7.27 cases per 1000 patient days) (Table 3). Among 972 cases with ICU-HAIs, 25 patients developed surgical site infections, and 15 patients developed non-catheter-associated urinary tract infections (CAUTI). Among 23,062 patients, 22, 804 (95.6\%) had catheters in place for a total 80,905 patient days; 199 (0.87\%) patients had CAUTI (0.85 cases per 100 patients or 1.38 cases per 1000 patient days). Among 14, 069 (59.0\%) patients in whom central lines were placed, 216 patients (1.54 cases per 100 patients, or 3.23 cases per 1000 patientdays) developed a CLABSI. In total, 21,792 (91.6\%) patients received invasive mechanical ventilation for 106, 173 ventilator days, of which 1540 patients developed VAEs (7.06 cases per 100 patients, or 14.5 cases per 1000 patient-days),

Table 2 Characteristics of Patients in the ICU-HAI Registry

\begin{tabular}{|c|c|c|c|c|c|c|c|}
\hline & GICU & SICU & NICU & RICU & TICU & PICU & Total \\
\hline \multicolumn{8}{|l|}{ Basic information } \\
\hline No. Patients & 4543 (19.7) & 4343 (I8.8) & $2033(8.8)$ & I338 (5.8) & 6407 (27.8) & $5183(22.5)$ & $23,062(100)$ \\
\hline No. Admissions & $4812(20.2)$ & $4422(18.5)$ & $2098(8.8)$ & I373 (5.8) & $6539(27.4)$ & $526 I(22.1)$ & $23,847(100)$ \\
\hline Men, n (\%) & $2946(64.8)$ & $2669(61.5)$ & $1106(54.4)$ & $907(67.8)$ & $3295(51.4)$ & $2788(53.8)$ & $|3,7| \mid(57.5)$ \\
\hline Age, year, median (QI-Q3) & $57(44,68)$ & $54(44,67)$ & $52(42,64)$ & $67(52,78)$ & $52(45,62)$ & I $(0,7)$ & $52(30-65)$ \\
\hline < 18 years, $n(\%)$ & $83(1.8)$ & $72(1.7)$ & $78(3.8)$ & $18(1.3)$ & $56(0.9)$ & & $4623(19.4)$ \\
\hline 18-44 years, n (\%) & $1072(23.6)$ & $1042(24.0)$ & $515(25.3)$ & $191(14.3)$ & $1480(23.1)$ & - & $4006(16.8)$ \\
\hline 45-64 years, n (\%) & I868 (4I.I) & $1948(44.9)$ & $943(46.4)$ & $392(29.3)$ & $3726(58.2)$ & - & $8793(36.9)$ \\
\hline$\geq 65$ years, $n(\%)$ & $1520(33.5)$ & $|28|(29.5)$ & $497(24.4)$ & $737(55.1)$ & 1145 (17.9) & - & $6425(26.9)$ \\
\hline \multicolumn{8}{|l|}{ Diagnosis } \\
\hline Valvulopathy & $3(0.1)$ & $0(0.0)$ & $0(0.0)$ & $4(0.3)$ & $3445(53.8)$ & $99(1.9)$ & 3551 (14.9) \\
\hline Heart septal defectsatrial & $2(0.0)$ & $0(0.0)$ & $0(0.0)$ & $0(0.0)$ & $507(7.9)$ & $1666(32.1)$ & $2175(9.1)$ \\
\hline Cerebral hemorrhage & $38(0.8)$ & $556(12.8)$ & $1009(49.6)$ & $35(2.6)$ & $\mathrm{I}(0.0)$ & $30(0.6)$ & $1669(7.0)$ \\
\hline Pneumonia & $5 I I(I I .2)$ & $22(0.5)$ & $24(1.2)$ & $824(61.6)$ & $33(0.5)$ & $38(0.7)$ & $1452(6.1)$ \\
\hline Aortic dissection & $38(0.8)$ & $160(3.7)$ & $0(0.0)$ & $\mathrm{I}(0.1)$ & $694(10.8)$ & $\mathrm{I}(0.0)$ & 894 (3.7) \\
\hline \multicolumn{8}{|l|}{ Outcome } \\
\hline ICU stays, median days (QI-Q3) & $6(2,14)$ & $4(2,8)$ & $8(4,8)$ & II $(6,19)$ & $3(2,4)$ & $4(3,7)$ & $4(3,8)$ \\
\hline Hospital stays, median days (QI-Q3) & $18(11,28)$ & $17(I 1,25)$ & $17(11,28)$ & $19(10,30)$ & $15(12,19)$ & $13(9,19)$ & $16(12,24)$ \\
\hline ICU mortality, n (\%) & $463(10.2)$ & $136(3.1)$ & $170(8.4)$ & $322(24.1)$ & $54(0.8)$ & $99(1.9)$ & $1244(5.4)$ \\
\hline Hospital mortality, n (\%) & $481(10.6)$ & $|5|(3.5)$ & $180(9.0)$ & $348(26.0)$ & $61(1.0)$ & $100(1.9)$ & $|32|(5.5)$ \\
\hline
\end{tabular}

Abbreviations: GICU, general intensive care unit; SICU, surgical intensive care unit; NICU, neurological intensive care unit, RICU, respiratory intensive care unit; TICU, thoracic surgery intensive care unit; PICU, pediatric intensive care unit. 
Table 3 Healthcare-Associated Infections Across ICUs

\begin{tabular}{|l|l|l|l|l|l|l|l|}
\hline & GICU & SICU & NICU & RICU & TICU & PICU & Total \\
\hline Person-time monitoring & 4844 & 4448 & 2108 & 1378 & 6563 & $527 I$ & 24,612 \\
Total ICU stays (days) & 57,106 & $35,20 I$ & 28,190 & 21,518 & 26,064 & 31,913 & 199,992 \\
HAI cases & 354 & 144 & 205 & 114 & 48 & 107 & 972 \\
Case-time infection (\%)* & 7.31 & 3.24 & 9.72 & 8.27 & 0.73 & 2.03 & 3.95 \\
Cases per 1000 patient-day (\%) & 6.20 & 4.09 & 7.27 & 5.30 & 1.84 & 3.35 & 4.86 \\
\hline
\end{tabular}

Notes: $*$ Case-time infection rates $=$ total number of cases developing $\mathrm{HAl} /$ total number of person-time monitoring; ${ }^{\#}$ Cases per 1000 patient-days $=$ total number of cases developing HAl/total number of ICU stays.

Abbreviations: GICU, general intensive care unit; SICU, surgical intensive care unit; NICU, neurological intensive care unit, RICU, respiratory intensive care unit; TICU, thoracic surgery intensive care unit; PICU, pediatric intensive care unit.

and 171 patients developed PVAP (0.78 cases per 100 patients, or 1.61 cases per 1000 patient days) (Table 4).

In the registry developed in the present study, 7780 (33.7\%) patients had various samples submitted for 60, 420 microbial cultures, of which 7773 (33.6\%) had bacterial cultures and $1594(6.9 \%)$ had fungal cultures. In addition, 3702 (16\%) patients had sputum cultures, 4932 (21.1\%) had blood cultures, and $962(4.2 \%)$ had urine culture (Figure 3). Overall, 5379 (23.1\%) patients had 23,235 microorganisms 1susceptibility tests. Among 855 patients who developed ICU-HAIs, 988 isolates were cultured, and the top three microorganisms were Acinetobacter baumannii (22.1\%), Klebsiella pneumoniae (15.4\%) and Enterococcus faecium $(9.2 \%)$.

\section{Quality of the ICU-HAI Registry}

The successful linkage of EMR, ICU system and ICU-HAI system using a unique identifier was $100 \%$. Validation of

Table 4 Healthcare-Associated Infections in the ICU-HAI Registry

\begin{tabular}{|l|l|l|l|}
\hline Catheterization & Ureter & $\begin{array}{l}\text { Central } \\
\text { Line }\end{array}$ & $\begin{array}{l}\text { Invasive } \\
\text { Mechanical } \\
\text { Ventilation }\end{array}$ \\
\hline $\begin{array}{l}\text { n (\%) } \\
\text { Patient-time } \\
\text { Patient-days }\end{array}$ & $\begin{array}{l}22,804(95.6) \\
147,408\end{array}$ & $\begin{array}{l}14,069(59.0) \\
14,460 \\
66,747\end{array}$ & $\begin{array}{l}21,792(91.6) \\
22,456 \\
106,173\end{array}$ \\
\hline HAI rate & CAUTI & CLABSI & PVAP \\
\hline $\begin{array}{l}\text { Case-time } \\
\text { infection (\%)* } \\
\text { Cases per 1000 } \\
\text { patient-days }\end{array}$ & $199(0.87)$ & $216(1.54)$ & $171(0.78)$ \\
\hline
\end{tabular}

Notes: *Case-time infection rates $=$ total number of cases developing catheter related infection/total number of patients placed catheterization; ${ }^{\#}$ Cases per 1000 patient-days $=$ total number of cases developing catheter related infection/total number of patient days of catheterization.

Abbreviations: $\mathrm{HAI}$, healthcare-associated infections; CAUTI, catheter-associated urinary tract infection; CLABSI, central line-associated bloodstream infection; PVAP, possible ventilator-associated pneumonia. data extraction and linkage by reviewing 240 randomly selected medical charts suggested that consistency with the original data was $100 \%$.

Evaluation of missing data showed that the completeness of ethnic and marital status records were $89.6 \%$ and $89.4 \%$ respectively. With respect to laboratory tests, more than $98 \%$ of patients had at least one important laboratory test, such as routine blood tests, serum glucose, serum creatinine, transaminases, coagulation function and lipid profiles. The median number of tests conducted was 4 to 5 per admission. Details of laboratory tests in the ICUHAI registry are summarized in Table 5. Validation of PVAP showed that $96.2 \%$ of the 158 PVAP cases were consistent with the evaluation of two infection control practitioners.

\section{Discussion}

We developed an ICU-HAI registry through successfully linking data from an EMR system, ICU system and ICUHAI system. To the best of our knowledge, this is the first registry to focus specifically on the management of HAIs in the ICU setting in China.

The registry has several important features. Firstly, it included a vast amount of data and information to adequately address important questions about HAIs. In total, the registry contained more than two hundred million rows of individual patient data from 23, 847 admissions. For each patient, the registry stored 492 variables, including many time-dependent variables (e.g. vital signs, prescriptions, and lab tests), a number that is even larger than that stored by other ICU databases, such as the Critical Care Health Informatics Collaborative (CCHIC) and the Intensive Care National Audit and Research Centre's Case Mix Programme (ICNARC CMP). ${ }^{23,24}$ Secondly, the collected data were complete and included all ICU patients, both those with, and those without HAIs. The HAIs in the ICU are complex, owing to a high proportion 


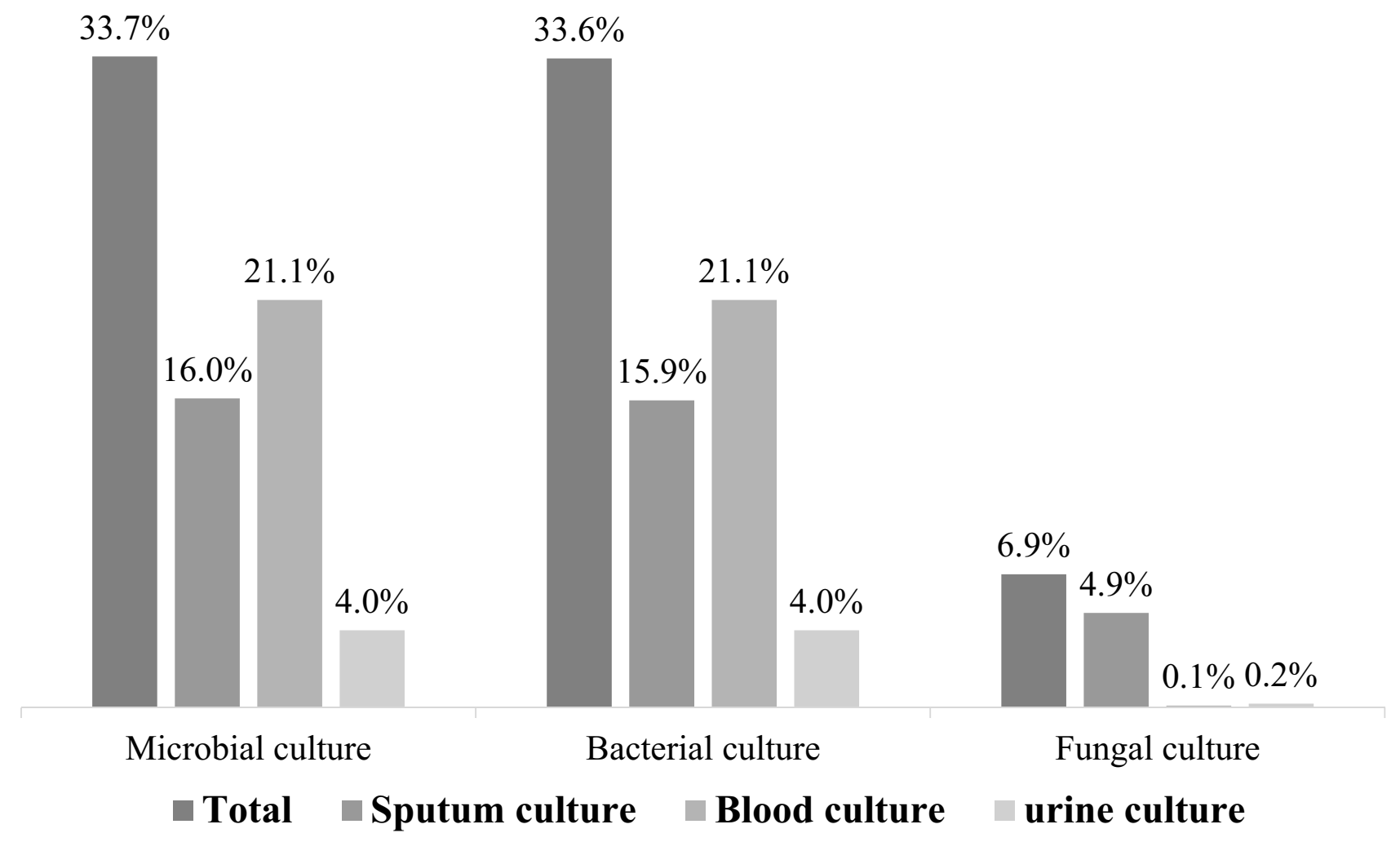

Figure 3 Microbial culture rates of patients included into the ICU-HAI registry.

of underlying diseases, multiple invasive devices and multidrug-resistant organisms. ${ }^{3,5}$ Studies investigating ICUHAIs often require complete documentation about the clinical care and outcomes of all patient admissions. However, traditional HAI surveillance registries usually collect data exclusively from patients with HAIs, and the collected information regarding clinical care is often limited. In contrast, the registry developed in the present study addresses these limitations by including all patients admitted to ICUs and achieving data linkage to develop a broader scope of the data. Thirdly, the data is validated, and its completeness and accuracy are generally high, particularly in key variables such as socio-demographics, laboratory tests, vital signs, and ICU-HAI related information. Because of these unique features, this registry would facilitate further investigations of many important questions regarding ICU-HAIs in China.

Nevertheless, our registry has limitations. Firstly, although the registry covers a large number of ICU admissions from three hospital facilities, the data comes from a homogeneous healthcare system, and the study setting may not be generalizable to all healthcare organizations across China. However, the registry used the best data sources currently available to address HAIs in the ICU setting in China. Secondly, we were unable to track the long-term outcomes of ICU patients after they were discharged from the hospital. We plan to develop linkage with the provincial HIS in the future.

\section{Comparison with Other Studies or Databases}

High-quality data sources for HAI research remain lacking. This is particularly the case for HAI research in critical care, a setting that is at high risk for HAIs. In critical care, one well-known database is the Medical Information Mart for Intensive Care (MIMIC-III), which is a freely accessible, single-center database located in the US. It has collected $70 \mathrm{~GB}$ of individual data related to patients admitted to the ICU and has supported research in critical care medicine. ${ }^{25-28}$ The CCHIC database is another multi-center longitudinal, linkable data resource, which has collected information from 11 adult ICUs at five UK teaching hospitals. ${ }^{23}$ This database includes the data of 18,074 patients and stores 258 variables. In comparison, our registry included a larger number of patients and variables than the CCHIC, and a larger volume of data than the MIMIC-III. More importantly, neither of the 
Table 5 Complement of Laboratory Test of the ICU-HAI Registry

\begin{tabular}{|l|l|l|l|}
\hline Key Variables & No. Admissions with Tests (\%) & Total Numbers of Test & Median Numbers of Tests/Admission \\
\hline White blood cell & $23,774(99.7)$ & 157,005 & $5(3,8)$ \\
Platelet & $23,774(99.7)$ & 157,003 & $5(3,8)$ \\
Hemoglobin & $23,774(99.7)$ & 157,000 & $5(3,8)$ \\
Direct bilirubin & $23,783(99.7)$ & 144,267 & $5(3,7)$ \\
Albumin & $23,783(99.7)$ & 144,093 & $5(3,7)$ \\
Serum creatinine & $23,783(99.7)$ & 144,436 & $4(3,7)$ \\
Serum natrium & $23,734(99.5)$ & 140,986 & $4(3,7)$ \\
Serum kalium & $23,734(99.5)$ & 140,986 & $4(3,7)$ \\
Triglyceride & $23,694(99.4)$ & 131,241 & $4(3,6)$ \\
Total cholesterol & $23,694(99.4)$ & 131,243 & $4(3,6)$ \\
Low density lipoprotein & $23,694(99.4)$ & 131,236 & $4(3,6)$ \\
Prothrombin time & $23,555(98.8)$ & 105,878 & $3(2,5)$ \\
International normalized ratio & $23,544(98.7)$ & 105,580 & $3(2,5)$ \\
D-dimer & $16,132(67.6)$ & 57,511 & $2(1,4)$ \\
Blood glucose & $23,780(99.7)$ & 143,857 & $5(3,7)$ \\
\hline Microbial culture & & & \\
Bacterial culture & $8007(33.6)$ & 57,212 & $(0,7)$ \\
Fungal culture & $1634(6.9)$ & 23,235 & $0(0,0)$ \\
Antimicrobial susceptibility testing & $5515(23.1)$ & & $0(0,0)$ \\
\hline
\end{tabular}

aforementioned databases collect information regarding HAIs; thus they both have little few applications in HAI research.

Previous studies regarding HAIs in the ICU setting also have limitations. Most employed small sample sizes and reported a very small number of events. ${ }^{13,29-32}$ For instance, one study, suggested no significant association between VAE and the timing of rehabilitation, included only 294 patients, among which 29 patients developed VAE. $^{30}$ Other larger studies, however, have been based on HAI surveillance data, from which information about non-infective patients are not usually included. ${ }^{14-16,33}$

More recently, two studies investigated strategies to prevent and control VAEs. ${ }^{17,18}$ Derived from a registry integrating multi-dimensional databases, with details about HAIs, one of the studies involving 9603 consecutive episodes of mechanical ventilation found that benzodiazepines and propofol were associated with an increased risk of VAE. ${ }^{17}$ The other study, which included 5539 consecutive patients undergoing mechanical ventilation found that head-of-bed elevation, sedative infusion interruptions, spontaneous breathing trials, and thromboembolism prophylaxis may be beneficial. ${ }^{18}$ In comparison, our registry involved a larger number of patients receiving consecutive mechanical ventilation and identified a greater number VAE cases. With the availability of a large volume of information regarding clinical care, our registry may add important knowledge to existing studies, particularly in the Chinese population.

\section{Conclusion}

In summary, we have successfully developed a large registry that specifically addresses HAIs in the ICU setting through integrating multi-source data with a high level of quality and comprehensiveness. We anticipate that this registry may bridge important knowledge gaps about infection control in the ICU setting both in China and worldwide.

\section{Acknowledgements}

This study was supported by the National Natural Science Foundation of China (Grant No. 71573183) and the 1.3.5 Project for Disciplines of Excellence, West China Hospital, Sichuan University (Grant No. ZYYC08003).

\section{Disclosure}

The authors have no conflicts of interest that are directly relevant to the content of this study.

\section{References}

1. Pittet D, Tarara D, Wenzel RP. Nosocomial bloodstream infection in critically ill patients. Excess length of stay, extra costs, and attributable mortality. JAMA. 1994;271(20):1598-1601. doi:10.1001/jama.1994.03 510440058033 
2. Vincent JL, Rello J, Marshall J, et al. International study of the prevalence and outcomes of infection in intensive care units. JAMA. 2009;302(21):2323-2329. doi:10.1001/jama.2009.1754

3. Alp E, Damani N. Healthcare-associated infections in intensive care units: epidemiology and infection control in low-to-middle income countries. J Infect Dev Ctries. 2015;9(10):1040-1045. doi:10.3855/ jidc. 6832

4. Zhu S, Kang Y, Wang W, et al. The clinical impacts and risk factors for non-central line-associated bloodstream infection in 5046 intensive care unit patients: an observational study based on electronic medical records. Crit Care. 2019;23(1):52. doi:10.1186/s13054-0192353-5

5. Marschall J, Mermel LA, Fakih M, et al. Strategies to prevent central line-associated bloodstream infections in acute care hospitals: 2014 update. Infect Control Hosp Epidemiol. 2014;35(7):753-771. doi:10.1086/676533

6. O'Grady NP, Alexander M, Burns LA, et al. Guidelines for the prevention of intravascular catheter-related infections. Clin Infect Dis. 2011;52(9):e162-193.

7. Huang EY, Chen C, Abdullah F, et al. Strategies for the prevention of central venous catheter infections: an American Pediatric Surgical Association Outcomes and Clinical Trials Committee systematic review. J Pediatr Surg. 2011;46(10):2000-2011. doi:10.1016/j. jpedsurg.2011.06.017

8. National Health and Family Planning commission. Regulation for prevention and control of healthcare associated infection in intensive care unit. 2017.

9. Magill SS, Klompas M, Balk R, et al. Developing a new, national approach to surveillance for ventilator-associated events: executive summary. Clin Infect Dis. 2013;57(12):1742-1746. doi:10.1093/cid/ cit577

10. Waters B, Muscedere J. A 2015 update on ventilator-associated pneumonia: new insights on its prevention, diagnosis, and treatment. Curr Infect Dis Rep. 2015;17(8):496. doi:10.1007/s119 08-015-0496-3

11. Lewis SC, Li L, Murphy MV, et al. Risk factors for ventilator-associated events: a case-control multivariable analysis. Crit Care Med. 2014;42(8):1839-1848. doi:10.1097/CCM.0000000 000000338

12. Liu J, Zhang S, Chen J, et al. Risk factors for ventilator-associated events: a prospective cohort study. Am J Infect Control. 2018;47 (7):744-749. doi:10.1016/j.ajic.2018.09.032

13. Ramirez-Estrada S, Lagunes L, Pena-Lopez Y, et al. Assessing predictive accuracy for outcomes of ventilator-associated events in an international cohort: the EUVAE study. Intensive Care Med. 2018;44 (8):1212-1220. doi:10.1007/s00134-018-5269-7

14. Magill SS, Li Q, Gross C, et al. Incidence and characteristics of ventilator-associated events reported to the national healthcare safety network in 2014. Crit Care Med. 2016;44(12):2154-2162. doi:10. 1097/CCM.0000000000001871

15. Zhu S, Cai L, Ma C, et al. The Clinical impact of ventilator-associated events: a prospective multi-center surveillance study. Infect Control Hosp Epidemiol. 2015;36(12):1388-1395. doi:10.1017/ice.2015.200

16. Venturini E, Montagnani C, Benni A, et al. Central-line associated bloodstream infections in a tertiary care children's University hospital: a prospective study. BMC Infect Dis. 2016;16(1):725. doi:10.1186/s12879-016-2061-6

17. Klompas M, Li L, Szumita P, et al. Associations between different sedatives and ventilator-associated events, length of stay, and mortality in patients who were mechanically ventilated. Chest. 2016;149 (6):1373-1379. doi:10.1378/chest.15-1389
18. Klompas M, Li L, Kleinman K, et al. Associations between ventilator bundle components and outcomes. JAMA Intern Med. 2016;176 (9):1277-1283. doi:10.1001/jamainternmed.2016.2427

19. West China Hospital of Sichuan University. About us. Available from: http://english.cd120.com/fastFacts/index.jhtml. Accessed December $11,2018$.

20. Centers for Disease Control and Prevention. Surveillance for ventilator-associated events. Available from: http://www.cdc.gov/ nhsn/acute-care-hospital/vae/index.html. Accessed March 25, 2014.

21. National Healthcare Safety Network (NHSN) of American. Patient Safety Component Manual (Bloodstream infection event). 2018. Available from: https://www.cdc.gov/nhsn/pdfs/validation/2018/ pcsmanual_2018-508.pdf. Accessed April 24, 2018 .

22. Li S, Yu C, Li Y, et al. Study design and baseline characteristics of inpatients with diabetes mellitus in a tertiary hospital in China: a database study based on electronic medical records. J Evid Based Med. 2018;11(3):152-157. doi:10.1111/jebm.12291

23. Harris S, Shi S, Brealey D, et al. Critical Care Health Informatics Collaborative (CCHIC): data, tools and methods for reproducible research: a multi-centre UK intensive care database. Int $\mathrm{J} \mathrm{Med}$ Inform. 2018;112:82-89. doi:10.1016/j.ijmedinf.2018.01.006

24. Harrison DA, Brady AR, Rowan K. Case mix, outcome and length of stay for admissions to adult, general critical care units in England, Wales and Northern Ireland: the Intensive Care National Audit \& Research Centre Case Mix Programme Database. Crit Care. 2004;8 (2):R99-R111. doi:10.1186/cc2834

25. Zhang Z, Zhu C, Mo L, et al. Effectiveness of sodium bicarbonate infusion on mortality in septic patients with metabolic acidosis. Intensive Care Med. 2018;44(11):1888-1895. doi:10.1007/s00134018-5379-2

26. Zhang Z, Hong Y, Liu N, et al. Association of do-not-resuscitate order and survival in patients with severe sepsis and/or septic shock. Intensive Care Med. 2017;43(5):715-717. doi:10.1007/s00134-0174690-7

27. Johnson AE, Pollard TJ, Shen L, et al. MIMIC-III, a freely accessible critical care database. Sci Data. 2016;3:160035. doi:10.1038/ sdata. 2016.35

28. Mayaud L, Lai PS, Clifford GD, et al. Dynamic data during hypotensive episode improves mortality predictions among patients with sepsis and hypotension. Crit Care Med. 2013;41(4):954-962. doi:10.1097/CCM.0b013e3182772adb

29. Garcia H, Romano-Carro B, Miranda-Novales G, et al. Risk factors for central line-associated bloodstream infection in critically ill neonates. Indian J Pediatr. 2019;86(4):340-346. doi:10.1007/s1209 8-019-02896-6

30. Shinoda T, Nishihara H, Shimogai T, et al. Relationship between ventilator-associated events and timing of rehabilitation in subjects with emergency tracheal intubation at early mobilization facility. Int J Environ Res Public Health. 2018;15(12):2892. doi:10.3390/ ijerph 15122892

31. Vaewpanich J, Akcan-Arikan A, Coss-Bu JA, et al. Fluid overload and kidney injury score as a predictor for ventilator-associated events. Front Pediatr. 2019;7:204. doi:10.3389/fped.2019.00204

32. Park SW, Ko S, An HS, et al. Implementation of central line-associated bloodstream infection prevention bundles in a surgical intensive care unit using peer tutoring. Antimicrob Resist Infect Control. 2017;6:103. doi:10.1186/s13756-017-0263-3

33. Hazamy PA, Van Antwerpen C, Tserenpuntsag B, et al. Trends in validity of central line-associated bloodstream infection surveillance data, New York State, 2007-2010. Am J Infect Control. 2013;41 (12):1200-1204. doi:10.1016/j.ajic.2013.06.006 


\section{Publish your work in this journal}

Clinical Epidemiology is an international, peer-reviewed, open access, online journal focusing on disease and drug epidemiology, identification of risk factors and screening procedures to develop optimal preventative initiatives and programs. Specific topics include: diagnosis, prognosis, treatment, screening, prevention, risk factor modification,

Submit your manuscript here: https://www.dovepress.com/clinical-epidemiology-journal systematic reviews, risk \& safety of medical interventions, epidemiology \& biostatistical methods, and evaluation of guidelines, translational medicine, health policies \& economic evaluations. The manuscript management system is completely online and includes a very quick and fair peer-review system, which is all easy to use. 